\title{
A Privacy-Masking Learning Algorithm for Online Distributed Optimization over Time-Varying Unbalanced Digraphs
}

\author{
Rong Hu $\mathbb{D D}^{1}$ and Binru Zhang $\mathbb{D}^{2}$ \\ ${ }^{1}$ School of Mathematics, Sichuan University of Arts and Science, Dazhou, Sichuan 635000, China \\ ${ }^{2}$ School of Finance and Economics, Yangtze Normal University, Fuling, Chongqing 408100, China \\ Correspondence should be addressed to Binru Zhang; 20130190034@uibe.edu.cn
}

Received 9 July 2021; Accepted 31 August 2021; Published 28 October 2021

Academic Editor: Jia-Bao Liu

Copyright ( $) 2021$ Rong Hu and Binru Zhang. This is an open access article distributed under the Creative Commons Attribution License, which permits unrestricted use, distribution, and reproduction in any medium, provided the original work is properly cited.

\begin{abstract}
This paper investigates a constrained distributed optimization problem enabled by differential privacy where the underlying network is time-changing with unbalanced digraphs. To solve such a problem, we first propose a differentially private online distributed algorithm by injecting adaptively adjustable Laplace noises. The proposed algorithm can not only protect the privacy of participants without compromising a trusted third party, but also be implemented on more general time-varying unbalanced digraphs. Under mild conditions, we then show that the proposed algorithm can achieve a sublinear expected bound of regret for general local convex objective functions. The result shows that there is a trade-off between the optimization accuracy and privacy level. Finally, numerical simulations are conducted to validate the efficiency of the proposed algorithm.
\end{abstract}

\section{Introduction}

Owing to high requirement of large scale and distributed data processing, distributed optimization (DO) is an attractive approach. The framework of DO permits multiple units to maintain their data unrevealed and optimize cooperatively a common optimization objective by local message exchanges and computations. As a consequence, DO helps to alleviate computational burden and strengthen both robustness to communication failures and scalability of data manipulation. In existing literature, distributed methods mainly include (sub)gradient-based approaches, dual averaging methods, primal-dual methods, and alternating direction method of multipliers (ADMM) based methods. We can refer to the recent survey and references therein [1].

Compared with classical DO in which the local objective functions are usually time-invariant, online distributed optimization (ODO) is applicable to the circumstances where local objective functions possibly change over time in some uncertain and even adversarial environments. Various ODO approaches have been increasingly developed in recent years $[3,4]$. In [2], Schwartz initially gave a review on the advancement of online convex optimization. In a static undirected graph, Yan et al. in [5] proposed a gradient-based online learning method for the ODO problem and established the regret bounds for convex cost functions. As an alternative, primal-dual-based method and its variants were further developed in $[6,7]$ for the ODO problems over undirected balanced networks. Very recently, Li et al. in [8] extended online mirror descent distributed algorithm [9] to a more general setup, in which a primal-dual mirror descent distributed method was proposed for solving the constrained ODO problems over a time-changing network with the requirement that the weight matrices are doubly stochastic.

All the distributed methods mentioned above are highly dependent on the demand of double-stochastic weight matrices induced by undirected or directed balanced graphs, which are fairly narrow and even unapplicable in practice. For example, a sensor network is directionally operated, since individual sensors possess diverse communication ranges limited by power supplies, which leads to the information flows between sensors become directed. 
Under this circumstance, the communication graphs with double stochasticity may be hard to be constructed. Recently, Nedić and Olshevsky in [10] initially introduced extra computations and communications to overcome the imbalance of networks by learning a specific eigenvector and then developed a push-sum-based algorithm. By using both column-stochastic and row-stochastic weight matrices simultaneously, $\mathrm{Pu}$ et al. [11] proposed an $\mathrm{AB}$ algorithm without learning the eigenvector. There are a lot of efforts for addressing unbalanced digraphs including the surplusbased algorithm [12], the epigraph-based algorithm [13], etc. Nevertheless, all these methods mainly focused on solving the DO problems. Akbari et al. [14] generalized the push-sum scheme to the online setup for the ODO problem over digraphs requiring column stochasticity of weight matrices. However, it requires that each node must acquire its out-degree for restructuring outgoing weights, which is difficult to be completed in a distributed scenario, especially when broadcast-based networks are adopted. In comparison with column-stochastic weight matrices, rowstochastic weight matrices are more practicable and easier to be satisfied, since each node can allocate these weights on messages by itself when received $[13,15,16]$.

However, the interactions among nodes in a distributed way will possibly lead to the disclosure of sensitive data, e.g., healthcare and financial accounts. As a consequence, data privacy has naturally aroused high attention when employing sensitive information. In distributed machine learning, there is an urgent need for designing DO methods while taking privacy-preserving property into account $[17,18]$. Recently, the work of [19] integrated homomorphic cryptography schemes into the DO method, resulting in strong privacy guarantees. However, the method encounters expensive computation and communication costs, which is inapplicable in large-scale networks. As a charming alternative, differential privacy (DP) has attracted considerable research interest due to its rigorous mathematical foundation and being executed easily. DP has become a well-known concept of privacy-preserving method and has found a lot of applications in practice [20]. The basic idea of DP methods is injecting artificial noises into true message, resulting in a balance between computation accuracy and privacy level. The recent work [21] proposed an ODO algorithm with DP over time-changing networks with weight-balancing digraphs. However, this method is only suitable for unconstrained problems, requiring that each node has the knowledge of its outdegrees.

All the limitations mentioned above motivate us to further explore an approach that has a stronger adaptability to more general applications. In this paper, by introducing the Laplace noise, we design an ODO algorithm with DP for solving constrained ODO problems in time-changing digraphs with row-stochastic matrices while preserving certain privacy. The main contributions of this article are summarized as follows:

(i) We design a differentially private online distributed method by injecting Laplace noises for addressing a constrained ODO problem over time-varying unbalanced digraphs. In contrast to existing privacypreserving methods in [22-24], our algorithm is suitable for more practical and general network topologies. More specifically, the network consists of time-varying unbalanced digraphs with rowstochastic matrices, which is much weaker than those in [22-24]. Furthermore, row stochasticity considered in this paper is more convenient and practical for each unit to allocate the weights on information it received than column stochasticity in $[21,25]$.

(ii) We establish a rigorous expected bound with a square-root regret for general convex local cost functions. The expected regret can achieve the same order of $O(\sqrt{T})$ as the methods in $[15,16]$ with no DP scheme, coinciding with best theoretical regrets obtained by state-of-the-art methods. Moreover, our analysis discloses a trade-off between optimization accuracy and privacy degree by adaptively adjusting the amount of Laplace noises injected.

(iii) In contrast to cloud-enabled distributed methods with differential privacy $[26,27]$, our algorithm is executed in a completely distributed fashion that individual node only communicates with its neighbors, without requiring a powerful cloud authority. Besides, our algorithm can overcome the infeasibility issue by introducing an additional projection step, whereas the algorithms proposed in $[22,23]$ possibly suffer from the issue.

The remainder of the paper is organized as follows. The problem of interest is first presented in Section 2. The algorithm is proposed in Section 3. The main results on DP and the regret bound are presented in Section 4. Section 5 provides numerical simulations. Finally, conclusions are given in Section 6.

Notation. Use $[N]=\{1, \ldots, N\}$ to represent the set of integers for a positive integer $N . x^{\top}$ denotes the transpose of a vector $x \in \mathbb{R}^{d}$. Use $e_{i}=\left(0, \ldots, 1_{i}, \ldots, 0\right)^{\top} \in \mathbb{R}^{d}$ to represent a base vector and $1_{d}=(1, \ldots, 1)^{\top} \in \mathbb{R}^{d}$ to represent a vector with all the entry equal to 1 . $[A]_{i j}$ stands for the $(i, j)$-th component of matrix $A$. A non-negative square matrix $A$ is row (column)-stochastic if each row (column) of $A$ sums up to 1 . The matrix $A$ is doubly stochastic if both each row and each column of $A$ sum up to 1 . The Euclidean projection operator is defined by $\Pi_{X}(x)=\operatorname{argmin}_{y \in X}\|y-x\|$ for any $x \in \mathbb{R}^{d}$, where $X$ is a convex set in $\mathbb{R}^{d}$. For a given parameter $\sigma>0$, we use $\operatorname{Lap}(\sigma)$ to represent the Laplace distribution. $\mathbb{P}(x)$ and $\mathbb{E}(x)$ denote the probability distribution and expectation of a random variable $x$, respectively.

\section{Problem Formulation and Preliminaries}

2.1. Graph Theory. Considering a network with $N$ nodes, we introduce a directed graph $\mathscr{G}=\{[N], \mathscr{E}\}$ to model the network communication among nodes, where $[N]$ is the node set and $\mathscr{E} \subseteq[N] \times[N]$ is the link set. We use $(j, i) \in \mathscr{E}$ 
to represent a directed link, implying that node $j$ directly transmits message to node $i$. Denote $N_{i}^{+}:=\{j \mid(j, i) \in \mathrm{E}\} \cup\{i\}$ and $N_{i}^{-}:=\{j \mid(i, j) \in \mathrm{E}\} \cup\{i\}$ by the collection of in-neighbors and out-neighbors for node $i$, respectively. A directed path is a sequence of directed links $\left(i_{1}, i_{2}\right),\left(i_{2}, i_{3}\right), \ldots,\left(i_{k-1}, i_{k}\right)$. The directed graph $\mathscr{G}$ is strongly connected if there exists at least a directed path from $i$ to $j$ for any $i, j \in[N]$. A weight matrix $A \in \mathbb{R}^{N \times N}$ is associated with the graph $\mathscr{G}$ with elements $[A]_{i j}>0$ if $(i, j) \in \mathscr{E},[A]_{i j}=0$ otherwise. The graph $\mathscr{G}$ is said to be balanced if $\sum_{j \in N_{i}^{+}}[A]_{i j}=$ $\sum_{j \in N_{i}^{-}}[A]_{j i}$ for all $i \in \mathscr{V}$, and unbalanced, otherwise. If the links of $\mathscr{G}$ change over time, we use $\mathscr{G}_{t}=\left\{[N], \mathscr{E}_{t}\right\}$ and $A_{t} \in \mathbb{R}^{N \times N}$ to denote the time-changing directed graphs and weight matrices, respectively.

Now we need the following assumptions on the directed graph sequence $\left\{\mathscr{G}_{t}\right\}_{t \geq 1}$.

Assumption 1. (a) The sequence of unbalanced graphs $\left\{\mathscr{G}_{t}\right\}_{t \geq 1}$ is uniformly jointly strongly connected, i.e., there exists a finite integer $B>0$ such that the graph $\mathscr{G}_{k} \cup \mathscr{G}_{k+1} \cup \cdots \cup \mathscr{G}_{k+B-1}$ is strongly connected for any $k \geq 0$. (b) At each time $t \geq 1$, the weight matrix $A_{t}$ is row-stochastic.

2.2. Differential Privacy. For two datasets $D:=\left\{d_{i}\right\}_{i \in[N]}$ and $D^{\prime}:=\left\{d_{i}^{\prime}\right\}_{i \in[N]}$, we say that $D$ and $D^{\prime}$ are adjacent if there is an $i \in[N]$ satisfying $d_{i} \neq d_{i}^{\prime}$, and $d_{j}=d_{j}^{\prime}$ for $\forall j \neq i$. We use $\operatorname{Adj}\left(D, D^{\prime}\right)$ to represent the adjacent relationship between $D$ and $D^{\prime}$, which means that $D$ and $D^{\prime}$ distinguish from one data point while all other data points are the same. For privacy concerns, we next introduce the concept of differential privacy.

Definition 1 ( $\varepsilon$-DP) (see [20]). For a given $\varepsilon>0$, a randomized method $\mathscr{M}$ preserves $\varepsilon$-differential privacy if for any adjacent datasets $D$ and $D^{\prime}$ and for any set of outputs $\Upsilon \subseteq R(\mathscr{M})$, the following relation holds:

$$
\mathbb{P}[\mathscr{M}(D) \in \Upsilon] \leq \mathbb{P}\left[\mathscr{M}\left(D^{\prime}\right) \in \Upsilon\right] \times \exp \left(\varepsilon \times\left|D \oplus D^{\prime}\right|\right),
$$

where $\oplus$ denotes the symmetric difference, $\left|D \oplus D^{\prime}\right|$ stands for the number of different records in $D$ and $D^{\prime}$, and $R(\mathscr{M})$ is the output range of $\mathscr{M}$.

By the concept of $\varepsilon$-DP, we can see that a small change of private dataset makes no remarkable difference on the output of $\mathscr{M}$. Hence, the adversary in the network obtains little information from participants. The parameter $\varepsilon$ measures the privacy level of $\mathscr{M}$, i.e., smaller $\varepsilon$ implies a higher degree of privacy-preserving property. Intuitively, $\varepsilon$ should be kept small. However, this possibly sacrifices the accuracy of the method $\mathscr{M}$. Thus, we had to seek a balance between the desired privacy degree and computation accuracy by carefully choosing $\varepsilon$.

For the sake of designing a differentially private mechanism, we hope to know the "sensitivity" of a randomized method $\mathscr{M}$. We now introduce the following definition of sensitivity, reflecting the supreme magnitude by which any single individual data changes the method $\mathscr{M}$ in the worst case.

Definition 2 (sensitivity) ( see [20]). At time $t$, the sensitivity of a randomized method $\mathscr{M}$ is defined by

$$
\Delta(t):=\sup _{D_{t}, D_{t}^{\prime}: \operatorname{Adj}\left(D_{t}, D_{t}^{\prime}\right)}\left\|\mathscr{M}\left(D_{t}\right)-\mathscr{M}\left(D_{t}^{\prime}\right)\right\|_{1}
$$

where $D_{t}$ and $D_{t}^{\prime}$ are the corresponding input datasets at time $t$.

2.3. Problem Formulation. In what follows, we formulate the distributed online problem. At time constant $t \in[T]$, each node $i \in[N]$ submits an action $x_{i, t}$ limited a common set $X \subseteq \mathbb{R}^{d}$, and then a local function $f_{i, t}: \mathbb{R}^{d} \longrightarrow \mathbb{R}$ is disclosed to this node and thereby incurred the cost $f_{i, t}\left(x_{i, t}\right)$. In this setting, the goal is to cooperatively optimize the global cost function at time $t \in[T]$ :

$$
f_{t}(x):=\sum_{i=1}^{N} f_{i, t}(x),
$$

subject to $x \in X$. It should be emphasized here that each node is accessible to its local function $f_{i, t}$ and the global objective $f_{t}$ is unavailable for any single node. Thus, all the nodes in the network need to exchange with each other for minimizing the global objective $f_{t}(x)$. Besides, each node's local function is disclosed before the node submits its action.

Due to the time-varying characteristics of cost functions, it is reburied to develop a method to decrease the gap between the cumulative payoff incurred by the method and the cost of the best fixed action in hindsight across a finite time period $T \geq 1$. That is, the total cost should be as close as possible to the best fixed action if all the information of $\left\{f_{i, t}\right\}_{t=1}^{T}, i \in[N]$ is known beforehand. Considering privacy concerns, all the nodes of the network only share their noisy actions with neighboring nodes based on a randomized strategy to guard private messages. Denote $\mathscr{F}_{t}$ by the $\sigma$-algebra produced by the history of a method until time $t$. The expected regret of node $j \in[N]$ is used to assess the performance of the method

$$
\mathbb{E}\left[R_{j}(T)\right]:=\sum_{t=1}^{T} \mathbb{E}\left[f_{t}\left(x_{j, t}\right)\right]-\sum_{t=1}^{T} f_{t}\left(x^{*}\right),
$$

where $x^{*}:=\operatorname{argmin}_{x \in X} \sum_{t=1}^{T} f_{t}(x)$ is the best fixed decision in hindsight. An online algorithm performs well if its regret is sublinear as a function of time period $T$.

The goal of this paper is to design a privacy-masking algorithm to minimize $f_{t}$ over time-changing generally unbalanced digraphs requiring that each node asymptotically drives its regret to zero over time in a distributed and online way. To the end, we introduce the following assumptions.

Assumption 2. (a) The constraint $X$ is a non-empty closed convex set. (b) For $i \in[N]$ and $t \geq 1$, local cost functions $f_{i, t}$ are convex on $X$. (c) For $i \in[N]$ and $t \geq 1$, the subgradient 
$\nabla f_{i, t}$ of $f_{i, t}$ is bounded on $X$, that is, there is a constant $G_{i}>0$ satisfying $\left\|\nabla f_{i, t}(x)\right\| \leq G_{i}$ for all $x \in X$. Let $G:=\max _{i \in[N]}$ $\left\{G_{i}\right\}$.

\section{Differentially Private Distributed Online Algorithm}

Now we present our algorithm. At time $t$, each node $i$ hold two variables $x_{i, t} \in \mathbb{R}^{d}$ and $w_{i, t} \in \mathbb{R}^{n}$. Firstly, node $i$ generates an artificially noise vector $n_{i, t}$, which is independent and identically distributed (i.i.d.) and drawn from the Laplace distribution $\operatorname{Lap}\left(M_{t}\right)$ with parameter $M_{t}$, and then the local action $x_{i, t}$ is perturbed by $n_{i, t}$ as follows:

$$
y_{i, t}=x_{i, t}+n_{i, t}
$$

After that, node $i$ shares the noisy version of its action with its out-neighbors $N_{i, t}^{-}$by

$$
z_{i, t}=\Pi_{X}\left(\sum_{j \in N_{i, t}^{-}}\left[A_{t}\right]_{i j} y_{j, t}\right),
$$

and then updates its action via an Euclidean projection using a stochastic subgradient $\widetilde{g}_{i, t}$ re-scaled by a number $w_{i i, t}$ as follows:

$$
x_{i, t+1}=\Pi_{X}\left(z_{i, t}-\eta_{t} \frac{\widetilde{g}_{i, t}}{w_{i i, t}}\right),
$$

where $\tilde{g}_{i, t}=\nabla f_{i, t}\left(z_{i, t}\right)+\delta_{i, t}$ is the stochastic subgradient of $f_{i, t}$ at $z_{i, t}, \delta_{i, t}$ is a random variable, and $\eta_{t}>0$ is the stepsize. Here $w_{i i, t}$ is the $i$-th entry of the auxiliary variable $w_{i, t}$, which is useful to balance the stochastic subgradient $\widetilde{g}_{i, t}$ due to the influence of unbalancedness of the network. The auxiliary variable $w_{i, t}$ is updated by

$$
w_{i, t+1}=\sum_{j \in N_{i, t}^{-}}\left[A_{t}\right]_{i j} w_{j, t} .
$$

Notice that making the projection onto $X$ in (6) is to maintain the point $z_{i, t}$ staying in $X$, since the function $\nabla f_{i, t}$ is well defined on $X$.

Now we summarize the above updates as in Algorithm 1.

Remark 1. When compared with existing algorithms, Algorithm 1 has the following features.

(i) The proposed algorithm is applicable to more general time-changing digraphs with row-stochastic weight matrices, whereas the algorithms are restrictive to a static undirected graph with doubly stochastic matrix $[22,23]$ or time-varying digraphs with column-stochastic matrices [25]. Our algorithm extends the method with a static digraph in [24] to time-varying digraphs with row-stochastic matrices; meanwhile, the choice of stepsize for our algorithm is adaptive, without knowing the time horizon $T$ in advance.

(ii) The algorithms proposed in [22, 23] possibly suffer from the infeasibility when using the subgradient $\nabla f_{i, t}$ to update, since large noise that provides high privacy guarantees can get the consensus update $\sum_{j \in N_{i, t}^{-}}\left[A_{t}\right]_{i j} y_{j, t}$ out of the convex domain $X$. Our algorithm can overcome this difficulty by introducing an additional projection step (see (2)).

(iii) By injecting an artificial and controllable noise $n_{i, t}$ to the accurate state $x_{i, t}$ at each iteration, the proposed algorithm can guard privacy. It can be viewed as an extension of distributed methods $[15,16]$ to the scenario that privacy concerns and online learning are simultaneously considered.

(iv) In contrast to cloud-enabled distributed methods with differential privacy $[26,27]$, our algorithm is executed in a completely distributed fashion that individual node only communicates with its neighbors, without requiring a powerful cloud authority.

Next we present a lemma that displays several properties of $A_{t}$ and the auxiliary variable $w_{i i, t}$, which are useful to analyze the performance analysis of Algorithm 1 .

Lemma 1 (see $[13,16])$. Suppose Assumption 1 holds. Let $A_{t: s}:=A_{s-1} \ldots A_{t}$ for $s \geq t$, where $A_{k: k}=I$ and $\left[A_{t: s}\right]_{i j}$ represents the element of $A_{t: s}$. Then, for any $t \geq 1$, there is a normalized vector $\pi_{t}\left(1_{N}^{\top} \pi_{t}=1\right)$ such that

(a) The relation $\left|\left[A_{t: s}\right]_{i j}-\pi_{j, t}\right| \leq \Gamma \gamma^{s-t}$ holds for all $s \geq t \geq 1$ and $i, j \in[N]$, with some constants $\Gamma>0$ and $\gamma \in(0,1)$.

(b) The relation $\omega^{-1} \leq \pi_{i, t} \leq 1$ holds for all $i \in[N]$, with a constant $\omega>0$.

(c) $\pi_{t}^{\top}=\pi_{t+1}^{\top} A_{t}$.

Due to introducing the noisy subgradients, we need some standard assumptions on the noise $\delta_{i, t}$.

Assumption 3. The stochastic noise $\delta_{i, t}, i \in[N], t \in[T]$ is independent and satisfies that $\mathbb{E}\left[\delta_{i, t} \mid \mathscr{F}_{t-1}\right]=0$ and $\mathbb{E}\left[\left\|\delta_{i, t}\right\| \mid \mathscr{F}_{t-1}\right] \leq v_{i}$ for a constant $v_{i}>0$.

Note that, under Assumptions 2 and 3, it holds that $\mathbb{E}\left[\widetilde{g}_{i, t} \mid \mathscr{F}_{t-1}\right]=\nabla f_{i, t}\left(z_{i, t}\right)$ and $\mathbb{E}\left[\left\|\widetilde{g}_{i, t}\right\| \mid \mathscr{F}_{t-1}\right] \leq G_{i}+v_{i}$. The stochastic noise $\delta_{i, t}, i \in[N], t \in[T]$ is also considered in $[10,11,21,25]$.

\section{Main Results}

In this section, we now give the main results including the $\varepsilon$-DP of Algorithm 1 and its expected regret bounds.

4.1. Differential Privacy Analysis. By Definition 2, the sensitivity $\Delta(t)$ grasps the magnitude of the worst output of an algorithm caused by the change of any single data. Hence, it is necessary to bound $\Delta(t)$ to derive the condition on noises for preserving $\mathcal{E}$-DP. Let $D_{t}$ be the input of Algorithm 1 at time $t$ that contains sensitive data required to be protected and each local cost function $f_{i, t}, \forall i \in[N]$, depends on $D_{t}$. Let $\left\{x_{i, t}\right\}_{i=1}^{N}, i \in[N]$ be the output sequence of Algorithm 1 


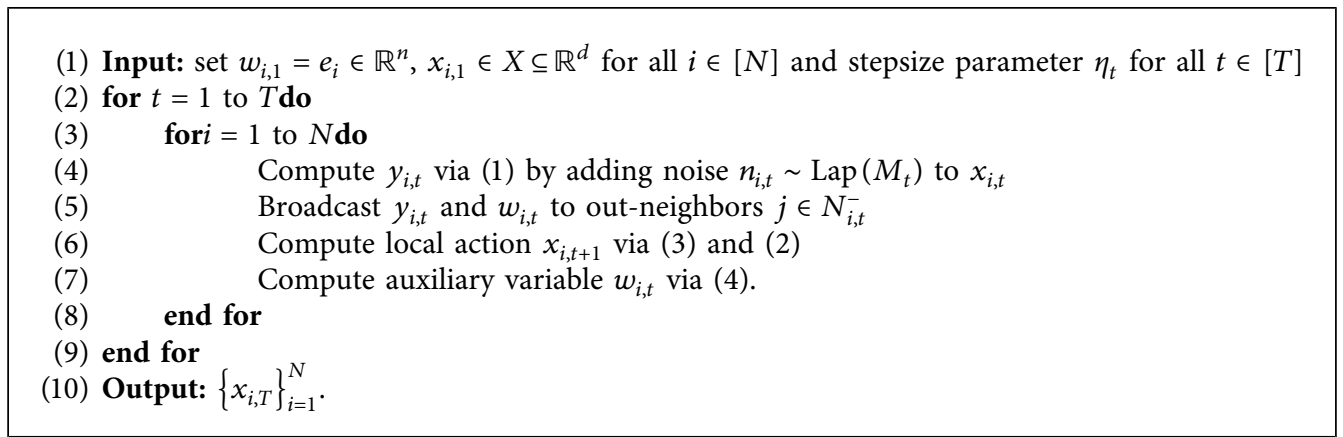

Algorithm 1: Differentially private distributed online algorithm using time-varying row-stochastic weight matrix.

at time $t$. We give a bound of $\Delta(t), \forall t \in[T]$ for Algorithm 1 in the following lemma.

Lemma 2. Let Assumptions 1-3 hold. For all $t \geq 1$, the sensitivity of Algorithm 1 is bounded as follows:

$$
\Delta(t) \leq 2 \sqrt{d} \omega \widehat{G} \eta_{t},
$$

where $d$ is the problem's dimension and $\widehat{G}:=\max _{i \in[N]}\left\{G_{i}+v_{i}\right\}$.

Proof. Let $D_{t}$ and $D_{t}^{\prime}$ be two adjacent datasets at time $t$ and $\left\{x_{i, t+1}\right\}$ and $\left\{x_{i, t+1}^{\prime}\right\}$ be the executions for Algorithm 1, respectively. Note that the adversary observes the output sequences kept the same, i.e., $z_{i, t}=z_{i, t}^{\prime}$. By Algorithm 1 and Definition 2, we get

$$
\begin{aligned}
\left\|\mathscr{M}_{t}(D)-\mathscr{M}_{t}\left(D^{\prime}\right)\right\|_{1} & =\left\|x_{i, t+1}-x_{i, t+1}^{\prime}\right\|_{1}=\left\|\Pi_{X}\left(z_{i, t}-\eta_{t} \frac{\tilde{g}_{i, t}\left(z_{i, t}\right)}{w_{i i, t}}\right)-\Pi_{X}\left(z_{i, t}^{\prime}-\eta_{t} \frac{\tilde{g}_{i, t}\left(z_{i, t}^{\prime}\right)}{w_{i, t}}\right)\right\|_{1} \\
& \leq \frac{\eta_{t}}{w_{i i, t}}\left\|\widetilde{g}_{i, t}\left(z_{i, t}\right)-\widetilde{g}_{i, t}\left(z_{i, t}^{\prime}\right)\right\|_{1} \\
& \leq \frac{\sqrt{d} \eta_{t}}{w_{i i, t}}\left\|\widetilde{g}_{i, t}\left(z_{i, t}\right)-\widetilde{g}_{i, t}\left(z_{i, t}^{\prime}\right)\right\| \\
& \leq \omega \sqrt{d} \eta_{t}\left(\left\|\widetilde{g}_{i, t}\left(z_{i, t}\right)\right\|+\left\|\widetilde{g}_{i, t}\left(z_{i, t}^{\prime}\right)\right\|\right),
\end{aligned}
$$

where the first inequality uses the non-expansive property of the Euclidean projection $\Pi_{X}$. From Assumptions 2 and 3, it yields that

$$
\mathbb{E}\left[\left\|\tilde{g}_{i, t}\right\| \mid \mathscr{F}_{t-1}\right] \leq G_{i}+v_{i} \leq \widehat{G} .
$$

From Definition 2, we further obtain

$$
\Delta(t) \leq \mathbb{E}\left[\left\|x_{i, t+1}-x_{i, t+1}^{\prime}\right\|_{1} \mid \mathscr{F}_{t}\right] \leq 2 \omega \sqrt{d} \widehat{G} \eta_{t},
$$

which implies the desired result.

Theorem 1. Under Assumptions 1-3, if we take the parameter $M_{t}=\Delta(t) / \varepsilon$ for all $t \geq 1$ when executing Algorithm 1 , then Algorithm 1 can ensure $\varepsilon$-differential privacy at the $t$-th iteration.

Proof. Let $x_{t}=\left[x_{1, t}, \ldots, x_{N, t}\right]^{\top}$ and $x_{t}^{\prime}=\left[x_{1, t}^{\prime}, \ldots, x_{N, t}^{\prime}\right]^{\top}$. By Lemma 2, we have

$$
\sum_{i=1}^{N} \sum_{l=1}^{d}\left|x_{i, t}^{l}-x_{i, t}^{\prime}\right|=\left\|x_{t}-x_{t}^{\prime}\right\|_{1} \leq \Delta(t)
$$
obtain 


$$
\begin{aligned}
\prod_{i=1}^{N} \prod_{l=1}^{d} \frac{\mathbb{P}\left[z_{i, t}^{l}-x_{i, t}^{l}\right]}{\mathbb{P}\left[z_{i, t}^{\prime l}-x_{i, t}^{\prime l}\right]} & =\prod_{i=1}^{N} \prod_{l=1}^{d} \frac{\exp \left(-\left|z_{i, t}^{l}-x_{i, t}^{l}\right| / M_{t}\right)}{\exp \left(-\left|z_{i, t}^{\prime l}-x_{i, t}^{\prime l}\right| / M_{t}\right)} \\
& \leq \prod_{i=1}^{N} \prod_{l=1}^{d} \exp \left(\frac{\left|z_{i, t}^{\prime l}-x_{i, t}^{\prime l}-z_{i, t}^{l}+x_{i, t}^{l}\right|}{M_{t}}\right) \\
& =\exp \left(\frac{\left\|x_{t}-x_{t}^{\prime}\right\|_{1}}{M_{t}}\right) \leq \exp \left(\frac{\Delta(t)}{M_{t}}\right),
\end{aligned}
$$

where the triangle inequality is used in the first inequality and Lemma 2 is used in the last inequality. By Definition 1, we have

$$
\mathbb{P}[\mathscr{M}(D) \in \Upsilon]=\prod_{t=1}^{T} \mathbb{P}\left[\mathscr{M}\left(D_{t}\right) \in \Upsilon\right] .
$$

From the preceding relations, we obtain

$$
\begin{aligned}
& \prod_{t=1}^{T} \mathbb{P}\left[\mathscr{M}\left(D_{t}\right) \in \Upsilon\right] \\
& \leq \prod_{t=1}^{T} \mathbb{P}\left[\mathscr{M}\left(D_{t}^{\prime}\right) \in \Upsilon\right] \times \prod_{t=1}^{T} \exp \left(\frac{\Delta(t)}{M_{t}} \times\left|D_{t} \oplus D_{t}^{\prime}\right|\right) \\
& =\prod_{t=1}^{T} \mathbb{P}\left[\mathscr{M}\left(D_{t}^{\prime}\right) \in \Upsilon\right] \times \exp \left(\frac{\Delta(t)}{M_{t}} \times\left|D_{t} \oplus D_{t}^{\prime}\right|\right) .
\end{aligned}
$$

Using the choice of the parameter $M_{t}$ and Definition 1, the proof is completed.

Theorem 1 shows that Algorithm 1 can guarantee $\varepsilon$-DP at each step by injecting a carefully designed Laplace noise. But it can only guard $\varepsilon$-DP at each single step. From the composition argument in [20], the privacy guarantee degrades after $T$-fold composition. The following corollary discloses the worst privacy guarantee after $T$ steps, which is at most the sum of the DP guarantee of each single step.

Corollary 1. Under the conditions of Theorem 1, Algorithm 1 can ensure TE-DP after $T$ iterations.

4.2. Regret Analysis. We analyze theoretically the regret bound of Algorithm 1. The following lemma provides the network disagreement error.

Lemma 3. Under Assumptions 1-3, the sequence $\left\{x_{i, t}\right\}$ is generated by Algorithm 1 taking $M_{t}=\Delta(t) / \varepsilon$. Then,

$$
\begin{aligned}
& \mathbb{E}\left[\left\|x_{i, t+1}-\bar{x}_{t+1}\right\| \mid \mathscr{F}_{t}\right] \\
& \leq \Gamma \gamma^{t} \sum_{j=1}^{N}\left\|\widetilde{x}_{j, 1}\right\|+2 \omega N \Gamma \widehat{G} \sum_{s=1}^{t-1} \gamma^{t-s} \eta_{s}+2 \omega \widehat{G} \eta_{t} \\
& \quad+2 \sqrt{2 d} N \Gamma \sum_{s=1}^{t-1} \gamma^{t-s} M_{s}+2 \sqrt{2 d} M_{t},
\end{aligned}
$$

where $\bar{x}_{t}:=\sum_{i=1}^{N} \pi_{i, t} x_{i, t}$ and constants $\Gamma$ and $\gamma$ are given in Lemma 1.

Proof. Let $x_{t}=\left[x_{1, t}, \ldots, x_{N, t}\right]^{\top}, u_{t}=\left[u_{1, t}, \ldots, u_{N, t}\right]^{\top}$ and $v_{t}=\left[v_{1, t}, \ldots, v_{N, t}\right]^{\top}$, where

$$
\begin{aligned}
u_{i, t} & =\Pi_{X}\left(z_{i, t}-\eta_{t} \frac{\tilde{g}_{i, t}}{w_{i i, t}}\right)-z_{i, t}, v_{i, t} \\
& =\Pi_{X}\left(\sum_{j=1}^{N}\left[A_{t}\right]_{i j} y_{j, t}\right)-\sum_{j=1}^{N}\left[A_{t}\right]_{i j} x_{j, t}, i \in[N] .
\end{aligned}
$$

Let $\bar{x}_{t}=\pi_{t}^{\top} x_{t}, \bar{u}_{t}=\pi_{t+1}^{\top} u_{t}, \bar{v}_{t}=\pi_{t+1}^{\top} v_{t}$. From (7) and (6), it gives rise to

$$
x_{t+1}=A_{t} x_{t}+v_{t}+u_{t} .
$$

Then, multiplying both sides of (19) by $1_{N} \pi_{t+1}^{\top}$ and making use of $\pi_{t}^{\top}=\pi_{t+1}^{\top} A_{t}$ from Lemma 1 , we get

$$
1_{N} \bar{x}_{t+1}=1_{N} \pi_{t}^{\top} x_{t}+1_{N} \bar{v}_{t}+1_{N} \bar{u}_{t} \text {. }
$$

Combining (19) and (20), we get

$x_{t+1}-1_{N} \bar{x}_{t+1}=\left(A_{t}-1_{N} \pi_{t}^{\top}\right) x_{t}+\left(v_{t}-1_{N} \bar{v}_{t}\right)+\left(u_{t}-1_{N} \bar{u}_{t}\right)$.

Letting $\quad \tilde{x}_{t+1}=x_{t+1}-1_{N} \bar{x}_{t+1}, \quad \widetilde{A}_{t}=A_{t}-1_{N} \pi_{t}^{\top}, \quad \tilde{v}_{t}=$ $v_{t}-1_{N} \bar{v}_{t}$, and $\tilde{u}_{t}=u_{t}-1_{N} \bar{u}_{t}$ and noting that $\left(A_{t}-1_{N} \pi_{t}^{\top}\right) 1_{N} \bar{x}_{t}=\left(A_{t} 1_{N}-1_{N} \pi_{t}^{\top} 1_{N}\right) \bar{x}_{t}=0$ due to row stochasticity of $A_{t}$, it follows from (21) that

$$
\begin{aligned}
\tilde{x}_{t+1}= & \widetilde{A}_{t} \tilde{x}_{t}+\widetilde{v}_{t}+\tilde{u}_{t} \\
= & \prod_{s=1}^{t} \widetilde{A}_{s} \widetilde{x}_{1}+\sum_{s=2}^{t}\left(\prod_{k=s}^{t} \widetilde{A}_{k}\right) \widetilde{v}_{s-1} \\
& +\tilde{v}_{t}+\sum_{s=2}^{t}\left(\prod_{k=s}^{t} \tilde{A}_{k}\right) \tilde{u}_{s-1}+\tilde{u}_{t} .
\end{aligned}
$$

By mathematical induction, it gives rise to $\prod_{k=s}^{t} \widetilde{A}_{k}=A_{t+1: s}-1_{N} \pi_{s}^{\top}$. Thus, relation (9) can be written as

$$
\begin{gathered}
\tilde{x}_{t+1}=\left(A_{t+1: 1}-1_{N} \pi_{1}^{\top}\right) \tilde{x}_{1}+\sum_{s=2}^{t}\left(A_{t+1: s}-1_{N} \pi_{s}^{\top}\right) \widetilde{v}_{s-1} \\
+\widetilde{v}_{t}+\sum_{s=2}^{t}\left(A_{t+1: s}-1_{N} \pi_{s}^{\top}\right) \widetilde{u}_{s-1}+\widetilde{u}_{t} .
\end{gathered}
$$


From the preceding relation, we can obtain

$$
\begin{aligned}
\tilde{x}_{i, t+1}= & \sum_{j=1}^{N}\left(\left[A_{t+1: 1}\right]_{i j}-\pi_{j, 1}\right) \tilde{x}_{j, 1} \\
& +\sum_{s=2}^{t} \sum_{j=1}^{N}\left(\left[A_{t+1: s}\right]_{i j}-\pi_{j, s}\right) \widetilde{v}_{j, s-1}+\widetilde{v}_{i, t} \\
& +\sum_{s=2}^{t} \sum_{j=1}^{N}\left(\left[A_{t+1: s}\right]_{i j}-\pi_{j, s}\right) \widetilde{u}_{j, s-1}+\widetilde{u}_{i, t} .
\end{aligned}
$$
have

Taking the norm on both sides of the above equality, we

$$
\begin{aligned}
\left\|x_{i, t+1}-\bar{x}_{t+1}\right\|= & \left\|\tilde{x}_{i, t+1}\right\| \\
\leq & \sum_{j=1}^{N}\left|\left[A_{t+1: 1}\right]_{i j}-\pi_{j, 1}\right|\left\|\widetilde{x}_{j, 1}\right\| \\
& +\sum_{s=2}^{t} \sum_{j=1}^{N}\left|\left[A_{t+1: s}\right]_{i j}-\pi_{j, s}\right|\left\|\widetilde{v}_{j, s-1}\right\|+\left\|\widetilde{v}_{i, t}\right\| \\
& +\sum_{s=2}^{t} \sum_{j=1}^{N}\left|\left[A_{t+1: s}\right]_{i j}-\pi_{j, s}\right|\left\|\widetilde{u}_{j, s-1}\right\|+\left\|\widetilde{u}_{i, t}\right\| .
\end{aligned}
$$

Using the non-expansivity of $\Pi_{X}$ and (18), we have

$$
\left\|u_{i, t}\right\| \leq \omega \eta_{t}\left\|\widetilde{g}_{i, t}\right\|,\left\|v_{i, t}\right\| \leq \sum_{j=1}^{N}\left[A_{t}\right]_{i j}\left\|n_{j, t}\right\| .
$$

From the definition of $\widetilde{u}_{i, t}$ and $\widetilde{v}_{i, t}$, we further obtain that $\mathbb{E}\left[\left\|\widetilde{u}_{i, t}\right\|\right] \leq 2 \omega \widehat{G} \eta_{t}$ and

$$
\mathbb{E}\left[\left\|\widetilde{v}_{i, t}\right\|\right] \leq \sum_{j=1}^{N}\left[A_{t}\right]_{i j} \mathbb{E}\left[\left\|n_{j, t}\right\|\right]+\sum_{i=1}^{N} \pi_{i, t} \mathbb{E}\left[\left\|n_{i, t}\right\|\right] .
$$

Using Lemma 1, Assumption 2, (24), and (27), we can get

$$
\begin{aligned}
\mathbb{E}\left[\left\|x_{i, t+1}-\tilde{x}_{t+1}\right\|\right] \leq & \Gamma \gamma^{t} \sum_{j=1}^{N}\left\|\tilde{x}_{j, 1}\right\| \\
& +2 \Gamma \sum_{s=2}^{t} \sum_{j=1}^{N} \gamma^{t-s+1} \sum_{i=1}^{N}\left[A_{s-1}\right]_{i j} \mathbb{E}\left[\left\|n_{i, s-1}\right\|\right] \\
& +\sum_{j=1}^{N}\left[A_{t}\right]_{i j} \mathbb{E}\left[\left\|n_{j, t}\right\|\right]+\sum_{i=1}^{N} \pi_{i, t} \mathbb{E}\left[\left\|n_{i, t}\right\|\right] \\
& +2 N \omega \Gamma \widehat{G} \sum_{s=2}^{t} \gamma^{t-s+1} \eta_{s-1}+2 \omega \widehat{G} \eta_{t} .
\end{aligned}
$$

Since $n_{i, t} \sim \operatorname{Lap}\left(M_{t}\right)$ and each entry of $n_{i, t} \in \mathbb{R}^{d}$ is independent, we obtain $\mathbb{E}\left[\left|n_{i, t}^{l}\right|^{2}\right]=2 M_{t}^{2}$, where $n_{i, t}^{l}$ is the $l$-th entry of $n_{i, t}$. Moreover, we have

$$
\begin{aligned}
& \mathbb{E}\left[\left\|n_{i, t}\right\|^{2}\right]=\sum_{l=1}^{d} \mathbb{E}\left[\left|n_{i, t}^{k}\right|^{2}\right] \leq 2 d M_{t}^{2}, \\
& \mathbb{E}\left[\left\|n_{i, t}\right\|\right] \leq \sqrt{\mathbb{E}\left[\left\|n_{i, t}\right\|^{2}\right]}=\sqrt{2 d} M_{t} .
\end{aligned}
$$

Thus, by taking the expectation condition on $\mathscr{F}_{t}$ for (28), the desired result can be obtained.

Lemma 4. Under Assumptions 1-3, the sequence $\left\{x_{i, t}\right\}$ is generated by Algorithm 1 with $M_{t}=\Delta(t) / \varepsilon$. Then, we have

$$
\begin{aligned}
& \sum_{i=1}^{N} \pi_{i, t+1} \mathbb{E}\left[\left\|x_{i, t+1}-x^{*}\right\|^{2} \mid \mathscr{F}_{t}\right] \\
& \leq \sum_{i=1}^{N} \pi_{i, t} \mathbb{E}\left[\left\|x_{i, t}-x^{*}\right\|^{2}\right]+2 d M_{t}^{2}+\widehat{G}^{2} \omega^{2} \eta_{t}^{2} \\
& \quad-B_{0} \eta_{t} \mathbb{E}\left[f_{t}\left(\bar{x}_{t}\right)-f_{t}\left(x^{*}\right)\right]+2 \sqrt{2 d} \omega G M_{t} \eta_{t} \\
& \quad+2 G \omega \eta_{t} \sum_{i=1}^{N} \pi_{i, t} \mathbb{E}\left[\left\|x_{i, t}-\bar{x}_{t}\right\|\right],
\end{aligned}
$$

where $B_{0}=2 \inf _{t \geq 1}\left\{\pi_{i, t+1} / w_{i i, t}\right\}$.

Proof. By (3) and the non-expansibility of $\Pi_{X}$, we obtain

$$
\begin{aligned}
& \mathbb{E}\left[\left\|x_{i, t+1}-x^{*}\right\|^{2} \mid \mathscr{F}_{t}\right] \\
& =\mathbb{E}\left[\left\|\Pi_{X}\left(z_{i, t}-\eta_{t} \frac{\tilde{\mathfrak{g}}_{i, t}}{w_{i i, t}}\right)-x^{*}\right\|^{2} \mid \mathscr{F}_{t}\right] \\
& \leq \mathbb{E}\left[\left\|z_{i, t}-\eta_{t} \frac{\tilde{g}_{i, t}}{w_{i i, t}}-x^{*}\right\|^{2}\right] \\
& =\mathbb{E}\left[\left\|z_{i, t}-x^{*}\right\|^{2}\right]-\frac{2 \eta_{t}}{w_{i i, t}} \mathbb{E}\left[\left\langle\widetilde{g}_{i, t}, z_{i, t}-x^{*}\right\rangle\right] \\
& +\frac{\eta_{t}^{2}}{w_{i i, t}^{2}} \mathbb{E}\left[\left\|\widetilde{g}_{i, t}\right\|^{2}\right] .
\end{aligned}
$$
that

Using the relation $\pi_{t}^{\top}=\pi_{t+1}^{\top} A_{t}$ from Lemma 1 , it follows

$$
\begin{aligned}
& \sum_{i=1}^{N} \pi_{i, t+1} \mathbb{E}\left[\left\|z_{i, t}-x^{*}\right\|^{2}\right] \\
& =\sum_{i=1}^{N} \pi_{i, t+1} \mathbb{E}\left[\left\|\Pi_{X}\left(\sum_{j=1}^{N}\left[A_{t}\right]_{i j}\left(x_{j, t}+n_{j, t}\right)\right)-x^{*}\right\|^{2}\right] \\
& \leq \sum_{i=1}^{N} \pi_{i, t+1} \mathbb{E}\left[\left\|\sum_{j=1}^{N}\left[A_{t}\right]_{i j}\left(x_{j, t}+n_{j, t}\right)-x^{*}\right\|^{2}\right] \\
& \leq \sum_{i=1}^{N} \pi_{i, t} \mathbb{E}\left[\left\|x_{i, t}+n_{i, t}-x^{*}\right\|^{2}\right] \\
& =\sum_{i=1}^{N} \pi_{i, t} \mathbb{E}\left[\left\|x_{i, t}-x^{*}\right\|^{2}\right]+\sum_{i=1}^{N} \pi_{i, t} \mathbb{E}\left[\left\|n_{i, t}\right\|^{2}\right] \\
& \leq \sum_{i=1}^{N} \pi_{i, t} \mathbb{E}\left[\left\|x_{i, t}-x^{*}\right\|^{2}\right]+2 d M_{t}^{2} .
\end{aligned}
$$

Combining (32) and (33) with Lemma 1, we obtain 


$$
\begin{aligned}
& \sum_{i=1}^{N} \pi_{i, t+1} \mathbb{E}\left[\left\|x_{i, t+1}-x^{*}\right\|^{2}\right] \\
& \leq \sum_{i=1}^{N} \pi_{i, t} \mathbb{E}\left[\left\|x_{i, t}-x^{*}\right\|^{2}\right]+2 d M_{t}^{2}+\widehat{G}^{2} \omega^{2} \eta_{t}^{2} \\
& \quad-\sum_{i=1}^{N} \pi_{i, t+1} \frac{2 \eta_{t}}{w_{i i, t}} \mathbb{E}\left[\left\langle\tilde{g}_{i, t}, z_{i, t}-x^{*}\right\rangle\right] .
\end{aligned}
$$

Note that

$$
\begin{aligned}
& \%\left\langle\widetilde{g}_{i, t}, z_{i, t}-x^{*}\right\rangle=\left\langle\nabla f_{i, t}\left(z_{i, t}\right), z_{i, t}-x^{*}\right\rangle \\
& +\left\langle\widetilde{g}_{i, t}-\nabla f_{i, t}\left(z_{i, t}\right), z_{i, t}-x^{*}\right\rangle \\
& -\left\langle\nabla f_{i, t}\left(z_{i, t}\right), z_{i, t}-x^{*}\right\rangle \leq f_{i, t}\left(x^{*}\right)-f_{i, t}\left(z_{i, t}\right) \\
& \leq f_{i, t}\left(x^{*}\right)-f_{i, t}\left(x_{t}\right)+G\left\|z_{i, t}-\bar{x}_{t}\right\| \\
& \leq f_{i, t}\left(x^{*}\right)-f_{i, t}\left(\bar{x}_{t}\right)+G\left\|\sum_{j=1}^{N}\left[A_{t}\right]_{i j}\left(x_{j, t}+n_{j, t}\right)-\bar{x}_{t}\right\| \\
& \leq f_{i, t}\left(x^{*}\right)-f_{i, t}\left(\bar{x}_{t}\right)+G \sum_{j=1}^{N}\left[A_{t}\right]_{i j}\left\|x_{j, t}+n_{j, t}-\bar{x}_{t}\right\| \text {. }
\end{aligned}
$$

Using (35) and (36) and the fact that $\mathbb{E}\left[\left\langle\tilde{g}_{i, t}-\right.\right.$ $\left.\left.\nabla f_{i, t}\left(z_{i, t}\right), z_{i, t}-x^{*}\right\rangle\right]=0$, we can estimate the third term in RHS of (34):

$$
\begin{aligned}
& -2 \eta_{t} \sum_{i=1}^{N} \frac{\pi_{i, t+1}}{w_{i i, t}} \mathbb{E}\left[\left\langle\widetilde{g}_{i, t}, z_{i, t}-x^{*}\right\rangle\right] \\
& \leq-2 \eta_{t} \sum_{i=1}^{N} \frac{\pi_{i, t+1}}{w_{i i, t}} \mathbb{E}\left[f_{i, t}\left(\bar{x}_{t}\right)-f_{i, t}\left(x^{*}\right)\right] \\
& \quad+2 G \eta_{t} \sum_{i=1}^{N} \frac{\pi_{i, t+1}}{w_{i i, t}} \sum_{j=1}^{N}\left[A_{t}\right]_{i j} \mathbb{E}\left[\left\|x_{j, t}+n_{j, t}-\bar{x}_{t}\right\|\right] .
\end{aligned}
$$

By letting $B_{0}=2 \inf _{t \geq 1}\left\{\pi_{i, t+1} / w_{i i, t}\right\}$, the first term of RHS in (37) can be bounded as follows:

$$
\begin{aligned}
& -2 \eta_{t} \sum_{i=1}^{N} \frac{\pi_{i, t+1}}{w_{i i, t}} \mathbb{E}\left[f_{i, t}\left(\bar{x}_{t}\right)-f_{i, t}\left(x^{*}\right)\right] \\
& \leq-B_{0} \eta_{t} \sum_{i=1}^{N} \mathbb{E}\left[f_{i, t}\left(\bar{x}_{t}\right)-f_{i, t}\left(x^{*}\right)\right] .
\end{aligned}
$$

For the second item in RHS of (37), we have

$$
\begin{aligned}
& 2 G \eta_{t} \sum_{i=1}^{N} \frac{\pi_{i, t+1}}{w_{i i, t}} \sum_{j=1}^{N}\left[A_{t}\right]_{i j} \mathbb{E}\left[\left\|x_{j, t}+n_{j, t}-\bar{x}_{t}\right\|\right] \\
& \leq 2 G \omega \eta_{t} \sum_{i=1}^{N} \pi_{i, t} \mathbb{E}\left[\left\|x_{i, t}-\bar{x}_{t}\right\|+\left\|n_{i, t}\right\|\right] .
\end{aligned}
$$

By substituting (38) and (39) into (37), it yields that

$$
\leq-B_{0} \eta_{t} \sum_{i=1}^{N} \mathbb{E}\left[f_{i, t}\left(\bar{x}_{t}\right)-f_{i, t}\left(x^{*}\right)\right]
$$

$$
\begin{aligned}
& -2 \eta_{t} \sum_{i=1}^{N} \frac{\pi_{i, t+1}}{w_{i i, t}} \mathbb{E}\left[\left\langle\widetilde{g}_{i, t}, z_{i, t}-x^{*}\right\rangle\right] \\
& +2 G \omega \eta_{t} \sum_{i=1}^{N} \pi_{i, t}\left(\mathbb{E}\left[\left\|x_{i, t}-\bar{x}_{t}\right\|\right]+\mathbb{E}\left[\left\|n_{i, t}\right\|\right]\right) .
\end{aligned}
$$

Finally, combining (34), (40), and (30), the desired result can be obtained.

Now we give the main result on convergence rate of Algorithm 1.

Theorem 2. Under Assumptions 1-3, the sequence $\left\{x_{i, t}\right\}$ is generated by Algorithm 1 with $M_{t}=\Delta(t) / \varepsilon$. If we take the stepsize as $\eta_{t}=1 / \sqrt{t}$ for all $t \geq 1$, then

$$
\mathbb{E}\left[R_{i}(T)\right] \leq B_{1}+B_{2}(\varepsilon) \sqrt{T},
$$

where $B_{1}=\Gamma G / 1-\gamma\left(N+2 \omega / B_{0}\right) \sum_{j=1}^{N}\left\|\tilde{x}_{j, 1}\right\|$ and $B_{2}(\varepsilon)=$ $N D^{2} / B_{0}+2 \widehat{G} \omega^{2} / B_{0}\left(8 d^{2} \widehat{G} / \varepsilon^{2}+\widehat{G}+4 \sqrt{2} d G / \varepsilon\right)+4 G \widehat{G}(N+$ $\left.2 \omega / B_{0}\right)(\omega+N \Gamma \gamma / 1-\gamma)(\omega+2 \sqrt{2} d / \varepsilon)$.

Proof. From the boundedness of subgradients of $f_{i, t}$, we have

$$
\begin{aligned}
f_{t}\left(x_{i, t}\right)-f_{t}\left(x^{*}\right) & =f_{t}\left(x_{i, t}\right)-f_{t}\left(\bar{x}_{t}\right)+f_{t}\left(\bar{x}_{t}\right)-f_{t}\left(x^{*}\right) \\
& \leq G \sum_{i=1}^{N}\left\|x_{i, t}-\bar{x}_{t}\right\|+f_{t}\left(\bar{x}_{t}\right)-f_{t}\left(x^{*}\right) .
\end{aligned}
$$

Summing up over $t=1, \ldots, T$ for the preceding inequality and then taking the expectation, we get

$$
\begin{aligned}
& \sum_{t=1}^{T} \mathbb{E}\left[f_{t}\left(x_{i, t}\right)-f_{t}\left(x^{*}\right)\right] \\
& \leq G \sum_{t=1}^{T} \sum_{i=1}^{N} \mathbb{E}\left[\left\|x_{i, t}-\bar{x}_{t}\right\|\right]+\sum_{t=1}^{T} \mathbb{E}\left[f_{t}\left(\bar{x}_{t}\right)-f_{t}\left(x^{*}\right)\right] .
\end{aligned}
$$

Rearranging the terms in (31) and then summing up from $t=1$ to $T$, it yields that 


$$
\begin{aligned}
& \sum_{t=1}^{T} \mathbb{E}\left[f_{t}\left(\bar{x}_{t}\right)-f_{t}\left(x^{*}\right)\right] \\
& \leq \sum_{t=1}^{T} \frac{1}{B_{0} \eta_{t}}\left(\sum_{i=1}^{N} \pi_{i, t} \mathbb{E}\left[\left\|x_{i, t}-x^{*}\right\|^{2}\right]\right. \\
& \left.\quad-\sum_{i=1}^{N} \pi_{i, t+1} \mathbb{E}\left\|x_{i, t+1}-x^{*}\right\|^{2}\right) \\
& \quad+\frac{2}{B_{0}} \sum_{t=1}^{T} \frac{M_{t}^{2}}{\eta_{t}}+\frac{\widehat{G}^{2} \omega^{2}}{B_{0}} \sum_{t=1}^{T} \eta_{t}+\frac{2 \sqrt{2 d} \omega G}{B_{0}} \sum_{t=1}^{T} M_{t} \\
& \quad+\frac{2 G \omega}{B_{0}} \sum_{t=1}^{T} \sum_{i=1}^{N} \pi_{i, t} \mathbb{E}\left[\left\|x_{i, t}-\bar{x}_{t}\right\|\right] .
\end{aligned}
$$

Let $P_{0}$ be the first term of RHS in (44). Now we estimate $P_{0}$ as follows:

$$
\begin{aligned}
P_{0} \leq & \frac{1}{B_{0} \eta_{1}} \sum_{i=1}^{N} \mathbb{E}\left[\left\|x_{i, 1}-x^{*}\right\|^{2}\right] \\
& +\sum_{t=2}^{T}\left(\frac{1}{B_{0} \eta_{t}}-\frac{1}{B_{0} \eta_{t-1}}\right) \sum_{i=1}^{N} \mathbb{E}\left[\left\|x_{i, t}-x^{*}\right\|^{2}\right] \leq \frac{N D^{2}}{B_{0} \eta_{T}},
\end{aligned}
$$

where we use the relation $0 \leq \pi_{i, t} \leq 1$ in the first inequality and $D^{2}=: \sup _{x, y \in X}\left\{\|x-y\|^{2}\right\}$ in the last inequality, respectively.

Combining (43)-(45) and Lemma 3, we have

$$
\begin{aligned}
& \sum_{t=1}^{T} \mathbb{E}\left[f_{t}\left(x_{i, t}\right)-f_{t}\left(x^{*}\right)\right] \\
& \leq \frac{N D^{2}}{B_{0} \eta_{T}}+\frac{2}{B_{0}} \sum_{t=1}^{T} \frac{M_{t}^{2}}{\eta_{t}}+\frac{\widehat{G}^{2} \omega^{2}}{B_{0}} \sum_{t=1}^{T} \eta_{t} \\
& \quad+\frac{2 \sqrt{2 d} \omega G}{B_{0}} \sum_{t=1}^{T} M_{t}+\frac{\Gamma G}{1-\gamma}\left(N+\frac{2 \omega}{B_{0}}\right) \sum_{j=1}^{N}\left\|\widetilde{x}_{j, 1}\right\| \\
& +2 N \omega \Gamma G \widehat{G}\left(N+\frac{2 \omega}{B_{0}}\right) \sum_{t=1}^{T} \sum_{s=1}^{t-2} \gamma^{t-s-1} \eta_{s} \\
& +2 \omega G \widehat{G}\left(N+\frac{2 \omega}{B_{0}}\right) \sum_{t=1}^{T} \eta_{t-1} \\
& +2 \sqrt{2 d} N \Gamma G\left(N+\frac{2 \omega}{B_{0}}\right) \sum_{t=1}^{T} \sum_{s=1}^{t-2} \gamma^{t-s-1} M_{s} \\
& +2 \sqrt{2 d} G\left(N+\frac{2 \omega}{B_{0}}\right) \sum_{t=1}^{T} M_{t-1} .
\end{aligned}
$$

Note that the choice of $\eta_{t}=1 / \sqrt{t}$ and $M_{t}=(\Delta(t) / \varepsilon) \leq(2 \sqrt{d} \widehat{G} \omega / \varepsilon) \eta_{t}$, and due to Lemma 2 , it follows from (46) that

$$
\begin{aligned}
& \sum_{t=1}^{T} \mathbb{E}\left[f_{t}\left(x_{i, t}\right)-f_{t}\left(x^{*}\right)\right] \\
& \leq \frac{\Gamma G}{1-\gamma}\left(N+\frac{2 \omega}{B_{0}}\right) \sum_{j=1}^{N}\left\|\widetilde{x}_{j, 1}\right\| \\
& \quad+\frac{N D^{2}}{B_{0}} \sqrt{T}+\frac{2 \widehat{G} \omega^{2}}{B_{0}}\left(\frac{8 d^{2} \widehat{G}}{\varepsilon^{2}}+\widehat{G}+\frac{4 \sqrt{2} d G}{\varepsilon}\right) \sqrt{T} \\
& +4 G \widehat{G}\left(N+\frac{2 \omega}{B_{0}}\right)\left(\omega+\frac{N \Gamma \gamma}{1-\gamma}\right)\left(\omega+\frac{2 \sqrt{2} d}{\varepsilon}\right) \sqrt{T}
\end{aligned}
$$

where we use the relation that $\sum_{t=1}^{T} 1 / \sqrt{t} \leq 2 \sqrt{T}$. This completes the proof.

Remark 2. From Theorem 1, the expected bound of regret for Algorithm 1 can be arrived at a square-root regret for convex cost functions. Notice that the expected regret has the same order of $O(\sqrt{T})$ as $[15,16]$ (with no differential privacy scheme) for a fixed $\varepsilon$ even when the Laplace noise is infused. In addition, the bound of regret is dependent on the connectivity of the network $\gamma$, the network size $N$, and the dimension $d$ of the underlying problem.

For a fixed $T$, the expected regret will increase (large enough) when the parameter $\varepsilon$ of privacy level becomes small. As seen from Theorem 2, the expected regret is of order $O\left(1 / \varepsilon^{2}\right)$ for any $\varepsilon \in(0,1)$. Thus, the results of Theorem 2 tell us that there is a comprehensive balance between the privacy degree and desired accuracy of algorithm.

\section{Numerical Simulations}

In this section, we present some numerical experiments to assess the performance of Algorithm 1.

We consider a network localization problem [25] with $N$ participating units. All the units in the network are used to coordinately detect a common vector $w \in \mathbb{R}^{d}$. At each time slot $t \in[T]$, unit $i$ has to access an observation vector $h_{i, t} \in R^{d_{i}}$, which changes over time with unknown noise due to environmental uncertainty. Assume that each unit $i$ has a linear model in a form as $h_{i}(w)=H_{i} w$, where $H_{i} \in \mathbb{R}^{d_{i} \times d}$ is a given observation matrix, and $H_{i} w=0$ if and only if $w=0$. The goal is to cooperatively seek the vector $\widehat{w} \in W \subseteq \mathbb{R}^{d}$ by minimizing the global cost function

$$
f(\widehat{w})=\sum_{t=1}^{T} \sum_{i=1}^{N} f_{i, t}(\widehat{w}),
$$

where $f_{i, t}(\widehat{w})=1 / 2\left\|h_{i, t}-H_{i} \widehat{w}\right\|^{2}$ is the local cost function of unit $i$. Here $h_{i, t}$ is the observation vector of unit $i$, modelled by $h_{i, t}=H_{i} w+\theta_{i, t}$, where $\theta_{i, t}$ denotes observation noise. Notice that each $f_{i, t}$ is time-varying and individual unit may 


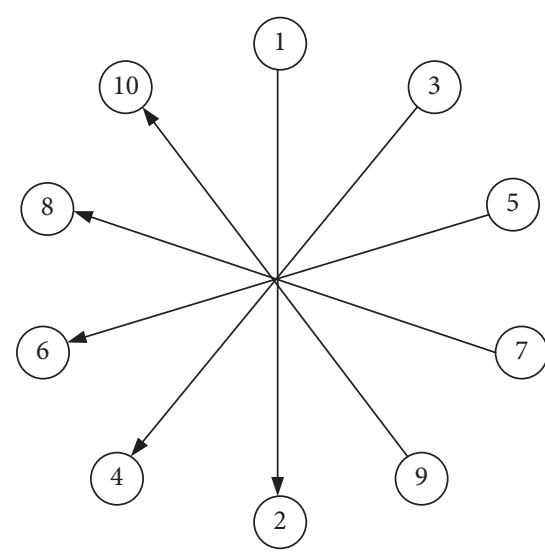

(a)
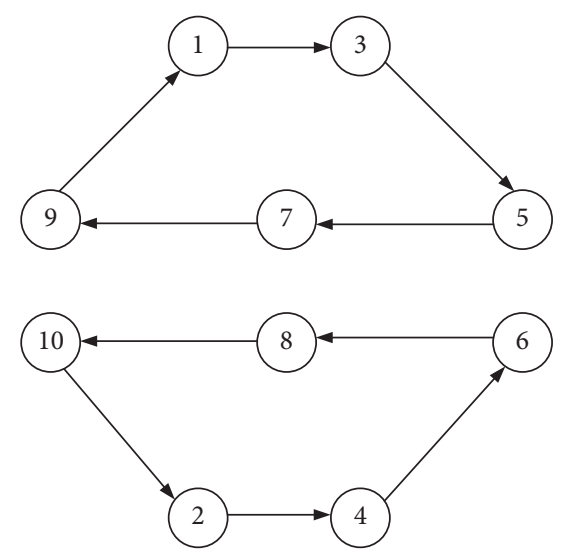

(b)

Figure 1: Two unbalanced directed graphs.
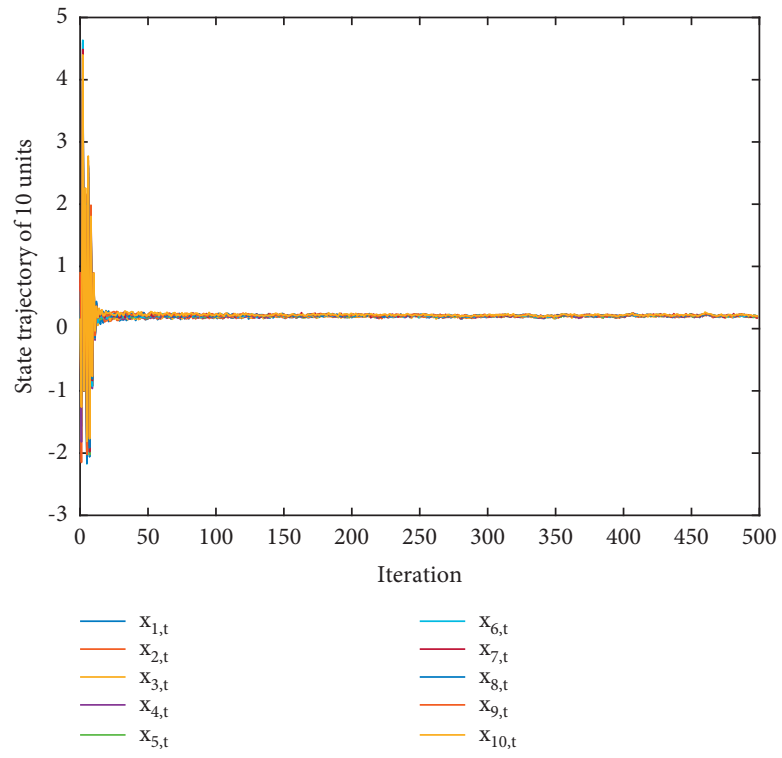

Figure 2: State trajectories of ten units.

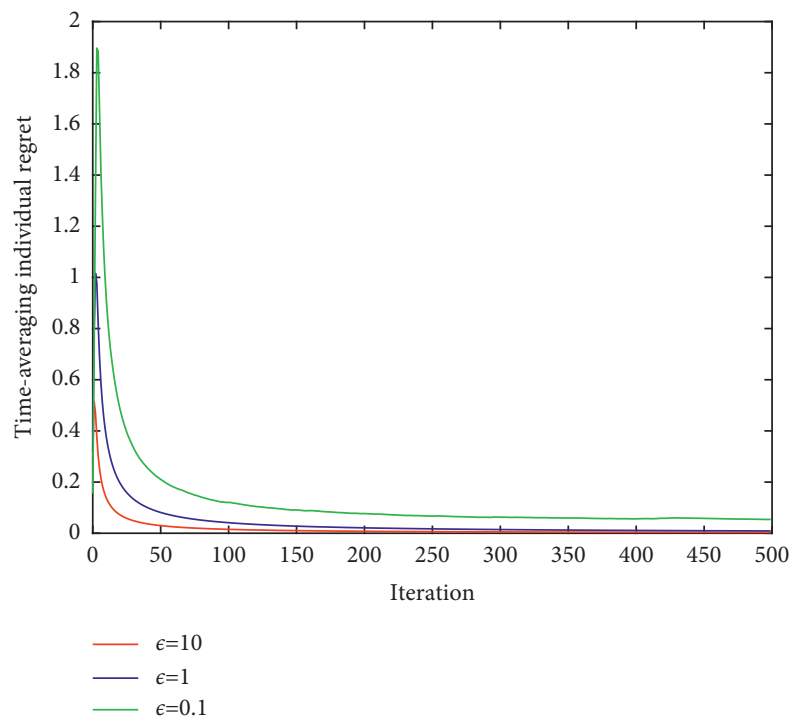

Figure 3: Evolutions of $R_{i}(T) / T$ under different levels of privacy. 


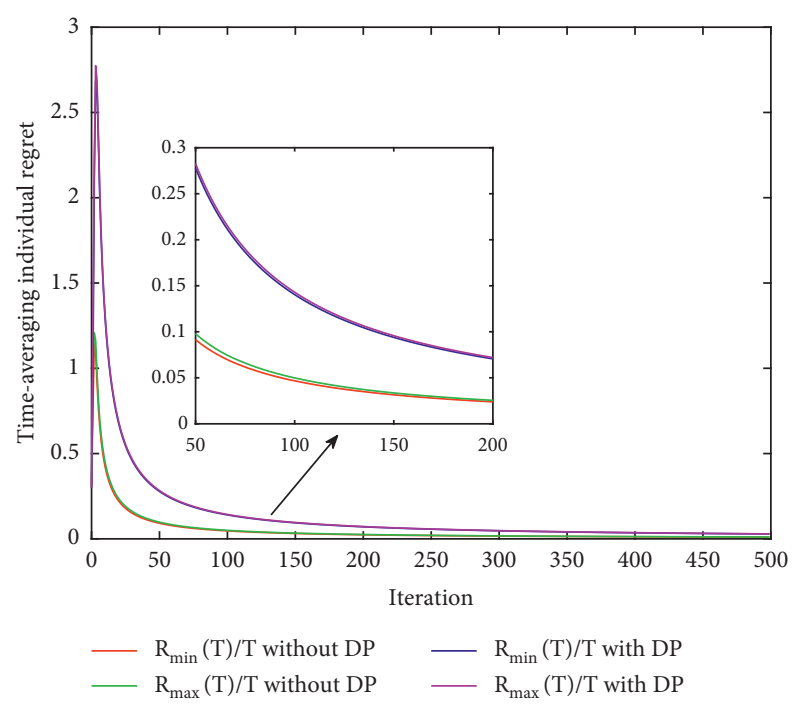

FIgure 4: Comparisons between Algorithm 1 with DP and the algorithm without DP in [16].

be unwilling to share its private data with other units. Thus, it requires to design an online distributed algorithm to commonly estimate a best vector $w$ while preserving the privacy of participants.

In simulations, we set $N=10$ and $d=1$. At time $t$, local cost function of unit $i$ is given by $f_{i, t}(\widehat{w})=1 / 2\left(h_{i, t}-H_{i} \widehat{w}\right)^{2}$, where $h_{i, t}=\alpha_{i, t} w+\beta_{i, t}$ and $H_{i} \in \mathbb{R}$. We set the constraint set $W=[-5,5]$ and let the coefficients $\alpha_{i, t}$ and $\beta_{i, t}$ randomly and uniformly draw from $(0,2]$ and $[-1,1]$ at each time $t$, respectively. Let $H_{i}$ be randomly generated from $(0,2]$ for each unit $i$ and set the stepsize $\eta_{t}=2 / t^{0.5}$. The communications among units are described in Figure 1. Note that any single graph is not strongly connected, but their jointed graphs are strongly connected. To satisfy Assumption 1, for each graph, we set the corresponding weight matrix by

$$
[A]_{i j}= \begin{cases}\frac{1}{\left|N_{i}^{i n}\right|}, & j \in N_{i}^{i n}, \\ 0, & \text { otherwise, }\end{cases}
$$

where $N_{i}^{\text {in }}$ can be numbered directly from Figure 1 . In the iteration process, we then randomly select a graph from the generated two graphs.

We use Algorithm 1 to estimate the vector $\widehat{w}$. The trajectories of ten units are illustrated in Figure 2 over earlier 500 iterations. It can be observed that the estimate of each unit $x_{i, t}$ can gradually arrive at the same value.

To study the effects of DP, we execute Algorithm 1 over the unbalanced digraphs described above with the stepsize $\eta_{t}=1 / t^{0.5}$ for three different levels of DP: $\varepsilon=10, \varepsilon=1$, and $\varepsilon=0.1$. The results are displayed in Figure 3, which show that our algorithm can achieve a sublinear regret and the parameter $\varepsilon$ determines a trade-off between the privacy level and the optimization accuracy. From this figure, we can observe that better privacy guarantees means worse accuracy of the algorithm.
Now we compare some numerical results between Algorithm 1 with DP by setting $\varepsilon=1$ and the proposed algorithm in [16] that does not consider DP. Figure 4 demonstrates the time-averaging individual regret $R^{j}(T) / T$ vs. iteration $T$ for two cases: one is the maximum time-averaging individual regret $\max R^{j}(T) / T$ and the other one is the minimum time-averaging individual regret $\min R^{j}(T) / T$. As seen in this figure, both maximum and minimum time-averaging individual regret is going to zero as the iteration $T$ grows up for both algorithms. Meanwhile, the maximum time-averaging individual regret is very closely the minimum one for both algorithms.

\section{Conclusion}

This paper has investigated a private distributed online constrained optimization in the setting that the unbalanced digraphs are time-varying requiring row stochasticity of weight matrices. By adopting the Laplace mechanism to protect sensitive data of participants, a differential privacy distributed online method was proposed. Under the convexity condition, the expected regret bounds of the proposed algorithm were derived. Finally, the trade-off between the privacy level and the algorithm accuracy is shown by numerical simulations.

\section{Data Availability}

The data used to support the findings of this study are available from the corresponding author upon request.

\section{Conflicts of Interest}

The authors declare that they have no conflicts of interest.

\section{Acknowledgments}

This research was supported by the Humanities and Social Science Fund of Ministry of Education of China under grant no. 20YJC630202 and by the Natural Science Foundation Projection of Chongqing CSTC under grant no. cstc2020jcyj-msxmX0287.

\section{References}

[1] A. Nedic, "Distributed gradient methods for convex machine learning problems in networks: distributed optimization," IEEE Signal Processing Magazine, vol. 37, no. 3, pp. 92-101, 2020.

[2] S. Shwartz, "Online learning and online convex optimization," Foundations and Trends in Machine Learningn, vol. 4, no. 2, pp. 107-194, 2012.

[3] J. Li, C. Li, W. Yu, X. Zhu, and X. Yu, "Distributed online bandit learning in dynamic environments over unbalanced digraphs," IEEE Transactions on Network Science and Engineering, vol. 1, 2021.

[4] B. Zhang, C. Gu, and J. Li, "Distributed convex optimization with coupling constraints over time-varying directed graphs $\dagger, "$ Journal of Industrial and Management Optimization, vol. 17, no. 4, pp. 2119-2138, 2021. 
[5] F. Yan, S. Sundaram, S. V. N. Vishwanathan, and Y. Qi, "Distributed autonomous online learning: regrets and intrinsic privacy-preserving properties," IEEE Transactions on Knowledge and Data Engineering, vol. 25, no. 11, pp. 24832493, 2013.

[6] A. Koppel, F. Y. Jakubiec, and A. Ribeiro, "A saddle point algorithm for networked online convex optimization," IEEE Transactions on Signal Processing, vol. 63, no. 19, pp. 51495164, 2015.

[7] D. Yuan, D. W. C. Ho, and G.-P. Jiang, “An adaptive primaldual subgradient algorithm for online distributed constrained optimization," IEEE Transactions on Cybernetics, vol. 48, no. 11, pp. 3045-3055, 2018.

[8] J. Li, C. Gu, Z. Wu, and T. Huang, "Online learning algorithm for distributed convex Optimization with time-varying coupled constraints and bandit feedback," IEEE Transactions on Cybernetics, vol. 18, pp. 1-12, 2020.

[9] S. Shahrampour and A. Jadbabaie, "Distributed online optimization in dynamic environments using mirror descent," IEEE Transactions on Automatic Control, vol. 63, no. 3, pp. 714-725, 2018.

[10] A. Nedic and A. Olshevsky, "Distributed optimization over time-varying directed graphs," IEEE Transactions on Automatic Control, vol. 60, no. 3, pp. 601-615, 2015.

[11] S. Pu, W. Shi, J. Xu, and A. Nedic, "Push-pull gradient methods for distributed optimization in networks," IEEE Transactions on Automatic Control, vol. 66, no. 1, pp. 1-16, 2021.

[12] C. Xi and U. A. Khan, "Distributed subgradient projection algorithm over directed graphs," IEEE Transactions on Automatic Control, vol. 62, no. 8, pp. 3986-3992, 2017.

[13] P. Xie, K. You, R. Tempo, S. Song, and C. Wu, "Distributed convex optimization with inequality constraints over timevarying unbalanced digraphs," IEEE Transactions on Automatic Control, vol. 63, no. 12, pp. 4331-4337, 2018.

[14] M. Akbari, B. Gharesifard, and T. Linder, "Distributed online convex optimization on time-varying directed graphs," IEEE Transactions on Control of Network Systems, vol. 4, no. 3, pp. 417-428, 2017.

[15] V. S. Mai and E. H. Abed, "Distributed optimization over directed graphs with row stochasticity and constraint regularity," Automatica, vol. 102, pp. 94-104, 2019.

[16] H. Li, Q. Lü, and T. Huang, "Distributed projection subgradient algorithm over time-varying general unbalanced directed graphs," IEEE Transactions on Automatic Control, vol. 64, no. 3, pp. 1309-1316, 2019.

[17] C. Zhang, M. Ahmad, and Y. Wang, "Admm based privacypreserving decentralized optimization," IEEE Transactions on Information Forensics and Security, vol. 14, no. 3, pp. 565-580, 2019.

[18] Y. Lou, L. Yu, S. Wang, and P. Yi, "Privacy preservation in distributed subgradient optimization algorithms," IEEE Transactions on Cybernetics, vol. 48, no. 7, pp. 2154-2165, 2018.

[19] Y. Lu and M. Zhu, "Privacy preserving distributed optimization using homomorphic encryption," Automatica, vol. 96, pp. 314-325, 2018.

[20] C. Dwork and A. Roth, "The algorithmic foundations of differential privacy," Foundations and Trends ${ }^{\circledR}$ in Theoretical Computer Science, vol. 9, no. 3-4, Article ID 211C407, 2014.

[21] J. Zhu, C. Xu, J. Guan, and D. O. Wu, "Differentially private distributed online algorithms over time-varying directed networks," IEEE Transactions on Signal and Information Processing over Networks, vol. 4, no. 1, pp. 4-17, 2018.
[22] Z. Huang, S. Mitra, and N. Vaidya, "Differentially private distributed optimization," International Conference on Distributed Computing Systems, vol. 4, pp. 1-10, 2015.

[23] C. Li, P. Zhou, L. Xiong, Q. Wang, and T. Wang, "Differentially private distributed online learning," IEEE Transactions on Knowledge and Data Engineering, vol. 30, no. 8, pp. 1440-1453, 2018.

[24] Y. Xiong, J. Xu, K. You, J. Liu, and L. Wu, "Privacy-preserving distributed online optimization over unbalanced digraphs via subgradient rescaling," IEEE Transactions on Control of Network Systems, vol. 7, no. 3, pp. 1366-1378, 2020.

[25] Q. Lü, X. Liao, T. Xiang, H. Li, and T. Huang, "Privacy masking stochastic subgradient-push algorithm for distributed online optimization," IEEE Transactions on Cybernetics, vol. 51, no. 6, pp. 3224-3237, 2021.

[26] M. T. Hale and M. Egerstedt, "Cloud-enabled differentially private multiagent optimization with constraints," IEEE Transactions on Control of Network Systems, vol. 5, no. 4, pp. 1693-1706, 2018.

[27] E. Nozari, P. Tallapragada, and J. Cortes, "Differentially private distributed convex optimization via functional perturbation," IEEE Transactions on Control of Network Systems, vol. 5, no. 1, pp. 395-408, 2018. 